\title{
Traveling Wave Solutions of Nonlinear Evolution Equations Via the Modified Simple Equation Method
}

\author{
A. K. M. Kazi Sazzad Hossain ${ }^{1, ~ *, ~ M . ~ A l i ~ A k b a r ² ~}$ \\ ${ }^{1}$ Department of Mathematics, Begum Rokeya University, Rangpur, Bangladesh \\ ${ }^{2}$ Department of Applied Mathematics, University of Rajshahi, Rajshahi, Bangladesh \\ Email address: \\ kazi_bru@yahoo.com (A. K. M. K. S. Hossain), ali_math74@yahoo.com (M. A. Akbar) \\ ${ }^{*}$ Corresponding author
}

\section{To cite this article:}

A. K. M. Kazi Sazzad Hossain, M. Ali Akbar. Traveling Wave Solutions of Nonlinear Evolution Equations Via the Modified Simple Equation Method. International Journal of Applied Mathematics and Theoretical Physics. Vol. 3, No. 2, 2017, pp. 20-25.

doi: 10.11648/j.ijamtp.20170302.11

Received: February 4, 2017; Accepted: February 21, 2017; Published: March 14, 2017

\begin{abstract}
Exact solutions of nonlinear evolution equations (NLEEs) are very crucial to realize the obscurity of many physical phenomena in mathematical science. The modified simple equation (MSE) method is especially effective and highly proficient mathematical instrument to obtaining exact traveling wave solutions to NLEEs arising in science, engineering and mathematical physics. Though the modified simple equation method effectively provides exact traveling wave solutions to nonlinear evolution equations for balance number less or equal 2 but the method did not applied to solve if the balance number is greater than 2. In this article, the MSE method is executed to construct exact traveling wave solutions of nonlinear evolution equations namely Kuramoto-Sivashinsky equation with balance number equal to 3 . The obtained solutions are expressed in terms of exponential and trigonometric functions including solitary and periodic solutions. Moreover the procedure of this method reduces huge volume of calculations.
\end{abstract}

Keywords: Modified Simple Equation (MSE) Method, Kuramoto-Sivashinsky Equation, Nonlinear Evolution Equations (NLEEs), Exact Traveling Wave Solutions

\section{Introduction}

The nonlinear evolution equations (NLEEs) are now extensively used in order to express the obscurity and inner mechanism of many physical phenomena in various fields of applied mathematics, science and engineering. Since nonlinear process are one of the most challenges and cannot be control easily because of its nonlinear characteristics hence NLEEs appear in a wide range of scientific research in various fields, such as nuclear physics, high energy physics, solid state physics, plasma physics, biomechanics, fluid mechanics, fluid dynamics, gas dynamics, optical fibers, elasticity, biochemistry, chemical reactions, geochemistry, meteorology etc. As a result, it is very essential to explore for promote exact traveling solutions to NLEEs for better realization. Consequently diverse groups of researchers have been working vigorously to develop effective methods for obtaining close form or exact solutions to NLEEs. That's why, recently several methods have been establish to explore exact solution, such as the nonlinear transform method [1], the functional variable method [2], the homogeneous balance method [3, 4], the direct algebraic method [5], the rank analysis method [6], the Jacobi-elliptic function expansion method [7], the complex hyperbolic function method [8], the tanh-function method [9], the inverse scattering transform [10], the Exp-function method [11-13], the sine-cosine method [14], the first integration method [15], the auxiliary parameter method [16], the Painleve expansion method [17], the Adomian decomposition method [18], the generalized Riccati equation method [19], the Lie group symmetry method [20], the modified Exp-function method [21], the perturbation method [22], the $\exp (-\Phi(\eta))$-expansion method [23-25], the ( $\left.G^{\prime} / G\right)$-expansion method [26-28], the asymptotic method [29], the improve $\left(G^{\prime} / G\right)$-expansion method [30], the modified simple equation method [31-34] etc. The recently developed modified simple equation method is getting popularity in use because of its straight forward calculation procedure but the method did not applied to solve if the balance number is greater than 2. To the best of our knowledge, till now only four or five articles are 
available in the literature concerning higher balance number (for balance number two) [32].

The objective of this article is to establish the modified simple equation method to construct fresh and more general exact traveling wave solutions to NLEEs namely KuramotoSivashinsky equation with balance number equal to 3 . The rest of the article is arranged as follows: In Section 2, modified simple equation method is discussed. In Section 3, the MSE method is applied to investigate the NLEEs indicated above. In Section 4, results are discussed and In Section 5 conclusions are provided.

\section{Outline of the Modified Simple Equation Method}

To explain the modified simple equation method, let us consider a nonlinear evolution equation in two independent variables $x$ and $t$ in the form:

$$
P\left(u, u_{t}, u_{x}, u_{t t}, u_{x x}, u_{x t}, \cdots\right)=0,
$$

where $u=u(x, t)$ is an unknown function and $P$ is a polynomial of $u(x, t)$ and its partial derivatives wherein the highest order derivatives and nonlinear terms are involved and the subscripts are used for partial derivatives. The essential steps of this method are presented in the following:

Step 1: Initiating a compound variable $\xi$, we combine the real variables $x$ and $t$ :

$$
u(x, t)=u(\xi), \xi=x \pm \omega t,
$$

where $\omega$ is the speed of the traveling wave.

The traveling wave transformations (2) allow us in reducing Eq. (1) into an ODE for $u=u(\xi)$ in the form:

$$
Q\left(\mathrm{u}, u^{\prime}, u^{\prime \prime}, u^{\prime \prime \prime} \ldots\right)=0,
$$

where $Q$ is a polynomial in $u(\xi)$ and its derivatives, the prime denotes the derivative with respect to $\xi$.

Step 2: Assume the solution of (3) can be expressed of the form:

$$
u(\xi)=\sum_{i=0}^{N} a_{i}\left(\frac{\psi^{\prime}(\xi)}{\psi(\xi)}\right)^{i}
$$

where $a_{i}(i=0,1,2,3, \ldots N)$ arearbitrary constants to be determined such that $a_{N} \neq 0$ and $\psi(\xi)$ is an unknown function to be evaluated later, such that $\psi^{\prime}(\xi) \neq 0$. The characteristic and uniqueness of this method is that, $\psi(\xi)$ is not known function or not a solution of any uncomplicated equation, whereas in the Exp-function method, tanh-function method, $\left(G^{\prime} / G\right.$ )-expansion method, sine-cosine method, Jacobi elliptic function method etc. the solution are proposed in terms of known function. Thus, it might be possible to obtain some new solution by this method.

Step 3: We determine the positive integerNarisesin (4) by balancing the highest order of linear and nonlinear terms appearing in (3).

Step 4: Compute the necessary derivatives $u^{\prime}, u^{\prime \prime}, \ldots$ and insert Eq. (4) into (3) and then we account the function $\psi(\xi)$. The above procedure makes a polynomial in $(1 / \psi(\xi))$. Equating the coefficients of same power of this polynomial to zero, yields a system of algebraic and differential equations that can be solved to get $a_{i}(i=0,1,2,3, \ldots N)$ and $\psi(\xi)$. This completes the determination of solutions of Eq. (1).

\section{Applications of the Method}

In this section, the modified simple equation method (MSE) has been applied to examine the closed form solutions leading to solitary wave solutions to the KuramotoSivashinsky equation. Let us consider the KuramotoSivashinsky equation in the form [27]:

$$
u_{t}=-u u_{x}-u_{x x}-u_{x x x x}
$$

This equation has applications in water waves and onedimensional evolution of small amplitude long waves in several problems arising in fluid dynamics.

The traveling wave transformations

$u(x, t)=u(\xi), \xi=x-\omega t$, where $\omega$ is a constant to be determined latter, reduce the Eq. (5) into an ODE of the form,

$$
u^{(i v)}+u^{\prime \prime \prime}+u u^{\prime}-\omega u^{\prime}=0
$$

Integrating (6) with respect to $\xi$ twice and the setting the constant of integration to zero, we can obtain the following result

$$
u^{\prime \prime \prime}+u^{\prime}+\frac{u^{2}}{2}-\omega u=0 .
$$

Balancing the order of nonlinear and linear terms $u^{2}$ and $u$ "' respectively, we obtain $N=3$

Therefore, the solution of Eq. (7) becomes,

$$
u(\xi)=a_{0}+a_{1}\left(\frac{\psi^{\prime}}{\psi}\right)+a_{2}\left(\frac{\psi^{\prime}}{\psi}\right)^{2}+a_{3}\left(\frac{\psi^{\prime}}{\psi}\right)^{3}
$$

where $a_{0}, a_{1}, a_{2}$ and $a_{3}$ are constants, such that $a_{3} \neq 0$ and $\psi(\xi)$ is an unknown function to be calculated. Substituting (8) and its derivatives into (7) and then equating the coefficients of $\psi^{0}, \psi^{-1}, \psi^{-2}, \psi^{-3}, \psi^{-4}, \psi^{-5}, \psi^{-6}$ to zero we achieveseven successive algebraic and differential equations,

$$
\begin{gathered}
\frac{a_{0}^{2}}{2}-\omega a_{0}=0 \\
-\omega a_{1} \psi^{\prime}+a_{0} a_{1} \psi^{\prime}+a_{1} \psi^{\prime \prime}+a_{1} \psi^{(i v)}=0
\end{gathered}
$$

$$
\begin{gathered}
2 a_{2} \psi^{\prime} \psi^{\prime \prime}+2 a_{2} \psi^{\prime} \psi^{(i v)}-4 a_{1} \psi^{\prime} \psi^{\prime \prime \prime}+a_{0} a_{2} \psi^{\prime 2}+6 a_{2} \psi^{\prime \prime} \psi^{\prime \prime \prime}-a_{1}^{2} \psi^{\prime 2}-3 a_{1} \psi^{\prime \prime 2}-\omega a_{2} \psi^{\prime 2}-3 a_{1} \psi \psi^{\prime \prime}+\frac{a_{1}^{2}}{2} \psi^{\prime 2}=0 \quad(11) \\
12 a_{1} \psi^{\prime 2} \psi^{\prime \prime}+3 a_{3} \psi^{\prime 2} \psi^{(i v)}+18 a_{3} \psi^{\prime} \psi^{\prime \prime} \psi^{\prime \prime \prime}+6 a_{3} \psi^{\prime \prime 3}-\omega a_{3} \psi^{\prime 3}+3 a_{3} \psi^{\prime 2} \psi^{\prime \prime}+a_{0} a_{3} \psi^{\prime 3}+a_{1} a_{2} \psi^{\prime 3}-2 a_{2} \psi^{3}- \\
14 a_{2} \psi^{\prime 2} \psi^{\prime \prime \prime}-24 a_{2} \psi^{\prime} \psi^{\prime \prime 2}=0
\end{gathered}
$$




$$
\begin{gathered}
-81 a_{3} a_{1}{\psi^{\prime}}^{2} \psi^{\prime \prime 2}-30 a_{3}{\psi^{\prime}}^{3} \psi^{\prime \prime \prime}-3 a \\
4 a_{3}{\psi^{\prime}}^{4} \psi^{\prime \prime}-24 a_{2} \psi^{\prime 5}+a_{2} a_{3} \psi^{\prime 5}=0 \\
-60 a_{3}{\psi^{\prime}}^{6}+\frac{a_{3}^{2}}{2} \psi^{\prime 6}=0
\end{gathered}
$$

From Eq. (9), it is found that $a_{0}=0$ and $a_{0}=2 \omega$.

And from Eq. (15), we achieve $a_{3}=120$, since $a_{3} \neq 0$.

From Eq. (14), it can be deduced that

$$
\frac{\psi^{\prime \prime}}{\psi^{\prime}}=\lambda
$$

Where

$$
\begin{array}{r}
\lambda=\frac{a_{2}\left(24-a_{3}\right)}{144 a_{3}} \quad \begin{array}{l}
\omega=-\frac{3}{361} \sqrt{209} \cdot \\
a_{0}, a_{1}, a_{2}, a_{3} \text { and } \omega \text { in }(8) \text { yields }
\end{array} \\
u(\xi)=\frac{-\frac{60}{361} \sqrt{209} c_{1}{ }^{3} e^{3 \lambda \xi / 2}+\frac{540}{361} \sqrt{209} c_{1}^{2} c_{2} \lambda e^{\frac{\lambda \xi}{2}-\frac{720}{361} \sqrt{209} c_{1} c_{2}{ }^{2} \lambda^{2} e^{-\lambda \xi / 2}}}{\left(c_{1} e^{\lambda \xi / 2}+c_{2} \lambda e^{-\lambda \xi / 2}\right)^{3}}
\end{array}
$$

Integrating (14) with respect to $\xi$, yields

$$
\psi^{\prime}=c_{1} \mathrm{e}^{\lambda \xi}
$$

And

$$
\psi=\frac{c_{1} \mathrm{e}^{\lambda \xi}}{\lambda}+c_{2}
$$

where $c_{1}$ and $c_{2}$ are arbitrary constants.

Case 1: When $a_{0}=0$ and $a_{3}=120$, solving Eq. (10)-(12) by using (17)-(19), provides $a_{1}=\frac{720}{19}, a_{2}=\frac{180}{19} \sqrt{209}$ and $\omega=-\frac{30}{361} \sqrt{209}$. Then by setting these values of where $\xi=(x-\omega t)$, and $\lambda=-\frac{\sqrt{209}}{19}$

Simplifying the exponential solution transformed to the trigonometric function in the close form solution of the equation (20) as

$$
\begin{gathered}
u(\xi)=\frac{-\frac{60}{361} \sqrt{209} c_{1}^{3}\left(\cos \left(\frac{3 \lambda \xi}{2}\right)+i \sin \left(\frac{3 \lambda \xi}{2}\right)\right)+A \cos \left(\frac{\lambda \xi}{2}\right)+i B \sin \left(\frac{\lambda \xi}{2}\right)}{\left(\left(c_{1}+c_{2} \lambda\right) \cos \left(\frac{\lambda \xi}{2}\right)+\left(c_{1}-c_{2} \lambda\right) i \sin \left(\frac{\lambda \xi}{2}\right)\right)^{3}}(21) \quad \begin{array}{l}
\text { obtain the following closed form solution of thashinsky equation: } \\
\text { Sivas }
\end{array} \\
u(\xi)=-\frac{\sqrt{209}}{722} \sec ^{2}\left(\frac{\lambda \xi}{2}\right)\left[\left(15 \cos \left(\frac{3 \lambda \xi}{2}\right) \sec \left(\frac{\lambda \xi}{2}\right)+45\right)+i\left(15 \sin \left(\frac{3 \lambda \xi}{2}\right) \sec \left(\frac{\lambda \xi}{2}\right)-315 \tan \left(\frac{\lambda \xi}{2}\right)\right)\right]
\end{gathered}
$$

where $\quad A=\frac{540}{361} \sqrt{209} c_{1}{ }^{2} c_{2} \lambda-\frac{720}{361} \sqrt{209} c_{1} c_{2}{ }^{2} \lambda^{2} \quad$ and $B=\frac{540}{361} \sqrt{209} c_{1}{ }^{2} c_{2} \lambda+\frac{720}{361} \sqrt{209} c_{1} c_{2}{ }^{2} \lambda^{2}$. Since $c_{1}$ and $c_{2}$ are integration constants, one may randomly pick their values, Therefore, if we set $c_{1}=1$ and $c_{2}=1 / \lambda$ in Eq. (21), we obtain the following closed form solution of the Kuramoto-

where $\xi=(x-\omega t)$, and $\lambda=-\frac{\sqrt{209}}{19}$

Again choosing $c_{1}=-1$ and $c_{2}=1 / \lambda$ the closed form solution (22) turn as

$$
u(\xi)=-\frac{\sqrt{209}}{722} \operatorname{cosec}^{2}\left(\frac{\lambda \xi}{2}\right)\left[\left(315-15 \sin \left(\frac{3 \lambda \xi}{2}\right) \operatorname{cosec}\left(\frac{\lambda \xi}{2}\right)\right)+i\left(15 \cos \left(\frac{3 \lambda \xi}{2}\right) \operatorname{cosec}\left(\frac{\lambda \xi}{2}\right)+45 \tan \left(\frac{\lambda \xi}{2}\right)\right)\right]
$$

Case 2: When $a_{0}=2 \omega, a_{1}=\frac{720}{19}, a_{2}=\frac{180}{19} \sqrt{209}, a_{3}=120$ and $\omega=\frac{30}{361} \sqrt{209}$, then (8) gives

$$
u(\xi)=\frac{60}{361} \sqrt{209}+\frac{-\frac{60}{361} \sqrt{209} c_{1}^{3} e^{3 \lambda \xi / 2}+\frac{540}{361} \sqrt{209} c_{1}^{2} c_{2} \lambda e^{\frac{\lambda \xi}{2}-\frac{720}{361} \sqrt{209} c_{1} c_{2}^{2} \lambda^{2} e^{-\lambda \xi / 2}}}{\left(c_{1} e^{\lambda \xi / 2}+c_{2} \lambda e^{-\lambda \xi / 2}\right)^{3}}
$$

where $\xi=(x-\omega t)$, and $\lambda=-\frac{\sqrt{209}}{19}$

Converting the exponential function into the trigonometric identity, the close form solution (24) becomes

$$
u(\xi)=\frac{60}{361} \sqrt{209}+\frac{-\frac{60}{361} \sqrt{209} c_{1}{ }^{3}\left(\cos \left(\frac{3 \lambda \xi}{2}\right)+i \sin \left(\frac{3 \lambda \xi}{2}\right)\right)+C \cos \left(\frac{\lambda \xi}{2}\right)+i D \sin \left(\frac{\lambda \xi}{2}\right)}{\left(\left(c_{1}+c_{2} \lambda\right) \cos \left(\frac{\lambda \xi}{2}\right)+\left(c_{1}-c_{2} \lambda\right) i \sin \left(\frac{\lambda \xi}{2}\right)\right)^{3}}
$$

Where $C=\frac{540}{361} \sqrt{209} c_{1}{ }^{2} c_{2} \lambda-\frac{720}{361} \sqrt{209} c_{1} c_{2}{ }^{2} \lambda^{2}$ and $D=\frac{540}{361} \sqrt{209} c_{1}{ }^{2} c_{2} \lambda+\frac{720}{361} \sqrt{209} c_{1} c_{2}{ }^{2} \lambda^{2}$ Setting $c_{1}=1$ and $c_{2}=1 / \lambda$ in Eq. (25), we obtain the following closed form solution of (25)

$$
u(\xi)=\frac{\sqrt{209}}{722}\left[120-\sec ^{2}\left(\frac{\lambda \xi}{2}\right)\left\{\left(15 \cos \left(\frac{3 \lambda \xi}{2}\right) \sec \left(\frac{\lambda \xi}{2}\right)+45\right)+i\left(15 \sin \left(\frac{3 \lambda \xi}{2}\right) \sec \left(\frac{\lambda \xi}{2}\right)-315 \tan \left(\frac{\lambda \xi}{2}\right)\right)\right\}\right]
$$

where $\xi=(x-\omega t)$, and $\lambda=-\frac{\sqrt{209}}{19}$ 
Again choose $c_{1}=-1$ and $c_{2}=1 / \lambda$ the closed form solution (25) turns as

$$
u(\xi)=\frac{\sqrt{209}}{722}\left[120+\operatorname{cosec}^{2}\left(\frac{\lambda \xi}{2}\right)\left\{\left(315-15 \sin \left(\frac{3 \lambda \xi}{2}\right) \operatorname{cosec}\left(\frac{\lambda \xi}{2}\right)\right)+i\left(15 \cos \left(\frac{3 \lambda \xi}{2}\right) \operatorname{cosec}\left(\frac{\lambda \xi}{2}\right)+45 \cot \left(\frac{\lambda \xi}{2}\right)\right)\right\}\right]
$$

\section{Results and Discussion}

In this section, we will discuss about the obtained solution of Kuramoto-Sivashinsky equation. Using the MSE method, achieve the traveling wave solutions from Eqs.(20) to (27). These solutions are general closed form traveling wave solutions which are soliton, and periodic wave solution respectively. From the above solution, the solutions (20) and (24) are represents in the exponential form where the solutions (21) to (23) and (25) to (27) are represents in terms of trigonometric functions. The solutions (22) and (26) are represents periodic wave solution and the solutions (23) and
(27) are represents singular soliton solutions. Solutions (22) to (23) and (26) to (27) are in the complex form, so the modulus and arguments of these solutions have been plotted. The graph of modulus and the arguments of (22) are plotted in the figure- 1 and figure- 2 respectively for $a_{0}=0, a_{1}=$ $\frac{720}{19}, a_{2}=\frac{180}{19} \sqrt{209}, a_{3}=120$, and $\omega=-\frac{30}{361} \sqrt{209}$ within the interval $-10 \leq x, t \geq-10$. The graph of modulus of (27) is singular soliton is shown in the figure- 3 for $a_{0}=2 \omega$, $a_{1}=\frac{720}{19}, a_{2}=\frac{180}{19} \sqrt{209}, a_{3}=120$, and $\omega=\frac{30}{361} \sqrt{209}$ within the interval $-10 \leq x, t \geq-10$. Others figures are omitted for convenience.

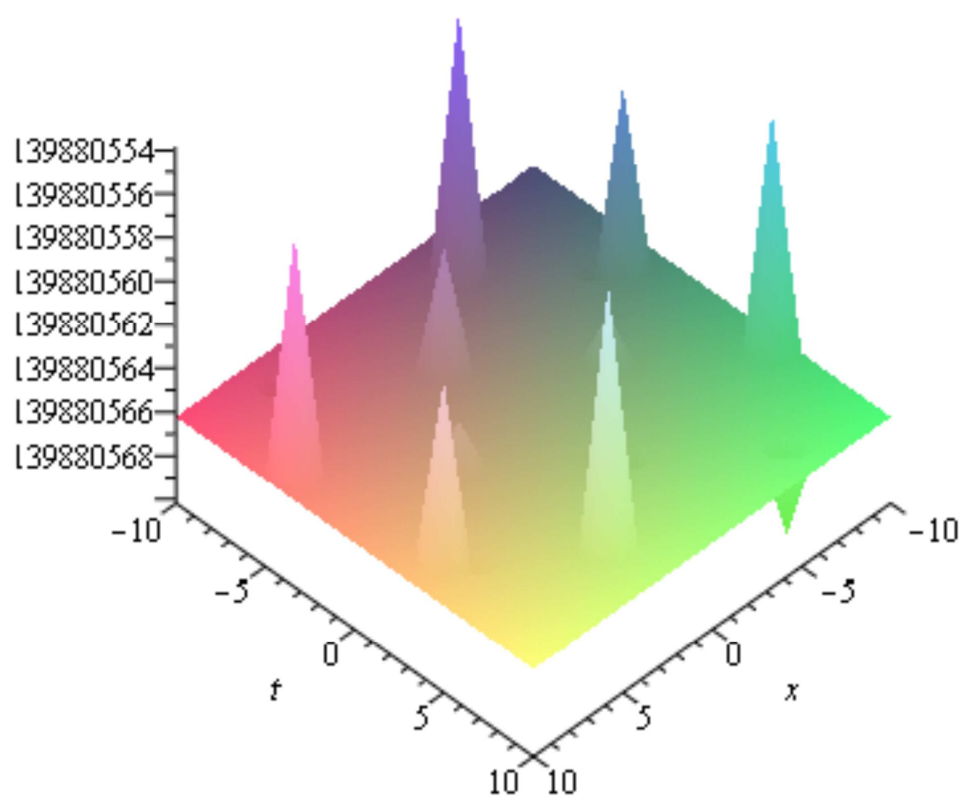

Figure 1. Plot of the modulus of solutionsu( $(\xi)$ in (22) of Kuramoto-Sivashinsky equation.

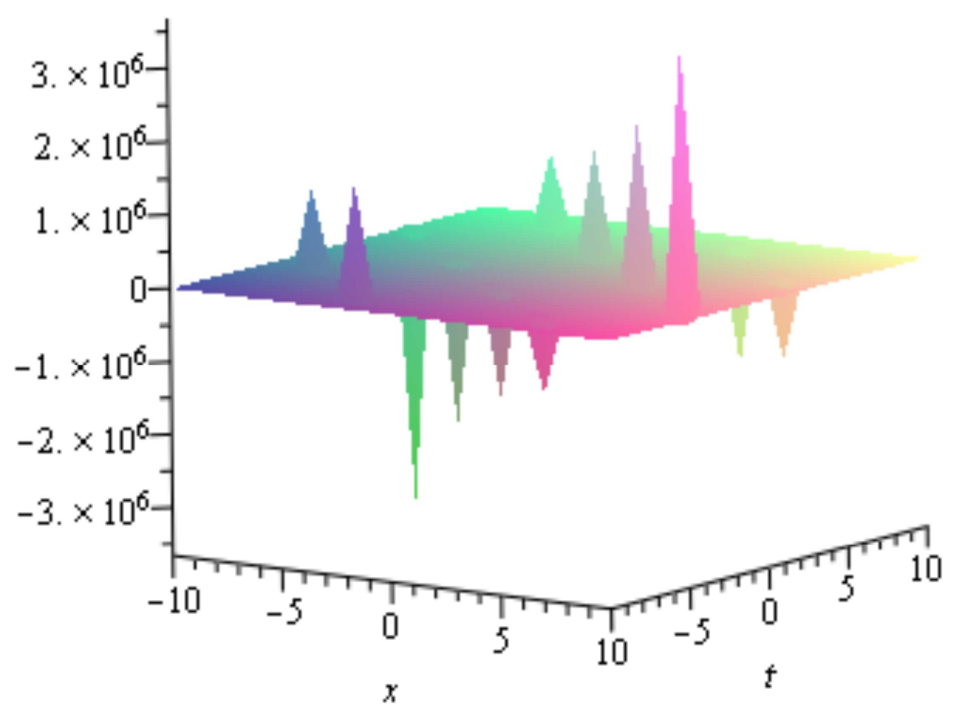

Figure 2. Plot of the argument of solutions $u(\xi)$ in (22) of Kuramoto-Sivashinsky equation. 


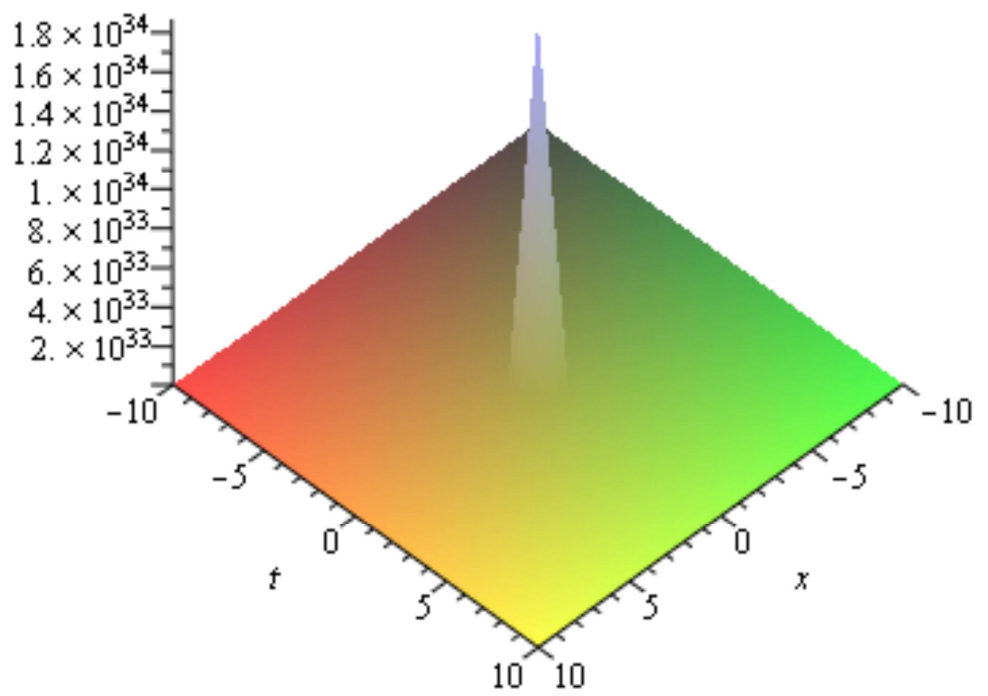

Figure 3. Plot of the modulus of solutions $u(\xi)$ in (27) of Kuramoto-Sivashinsky equation.

\section{Conclusion}

In this article, the modified simple equation method has been successfully used to find the exact traveling wave solutions of Kuramoto-Sivashinsky equation. The solutions are verified to check the correctness of the solutions by putting them back into the original equation and found correct. It is significant to observe that, here, we obtained the value of the coefficients $a_{0}, a_{2}$ etc without using any symbolic computation software such as Maple, Mathematica, etc. Therefore this method is very easy and straightforward to handling. Also it is quite capable and can be applied for finding exact solutions of other NLEEs in mathematical physics.

\section{References}

[1] L. Yang, J. Liu, K. Yang, Exact solutions of nonlinear PDE nonlinear transformation and reduction of nonlinear PDE to a quadrature, Phys. Lett. A, 278 (2001) 267-270.

[2] A. C. Cevikel, A. Beker, M. Akar, S. San, A procedure to construct exact solution of nonlinear evolution equations, Pramana J. Phys., 79(3) (2012) 337-344.

[3] E. M. E. Zayed, H. A. Zedan, K. A. Gepreel, On the solitary wave solutions for nonlinear Hirota-Sasuma coupled KDV equations, Chaos, Solitons Fract., 22 (2004) 285-303R. Hirota, Exact envelope soliton solutions of a nonlinear wave equation, J. Math. Phys., 14(1973) 805-810.

[4] M. Wang, Solitary wave solutions for variant Boussinesq equations, Phy. Lett. A, 199 (1995) 169-172.

[5] W. Hereman, P. P. Banerjee, A. Korpel, G. Assanto, A. Van Immerzeele, A. Meerpoel, Exact solitary wave solutions of nonlinear evolution and wave equations using a Direct algebraic method, J. Phys. A: Math. General 19 (5) (1986) 607-628.

[6] X. Feng, Exploratory approach to explicit solution of nonlinear evolutions equations, Int. J. Theor. Phys. 39 (2000)
207-222. Liu S., Fu Z., Liu S. D. and Zhao Q., Jacobi elliptic function expansion method and periodic wave solutions of nonlinear equations. Phys. Lett. A. 289 (2001) 69-74.

[7] Liu S., Fu Z., Liu S. D. and Zhao Q., Jacobi elliptic function expansion method and periodic wave solutions of nonlinear equations. Phys. Lett. A. 289 (2001) 69-74.

[8] M. L. Wang, Y. B. Zhou, The periodic wave equations for the Klein Gordon Schordinger equations, Phys. Lett. A, 318 (2003) 84-92.

[9] H. A. Nassar, M. A. Abdel-Razek, A. K. Seddeek, Expanding the tanh-function method for solving nonlinear equations, Appl. Math., 2 (2011) 1096-1104.

[10] M. J. Ablowitz, P. A. Clarkson, Soliton, Nonlinear Evolution Equations and Inverse Scattering, Cambridge University Press, New York, 1991.

[11] A. Bekir, A. Boz, Exact solutions for nonlinear evolution equations using Exp-function method, Phy. Lett. A, 372 (2008) 1619-1625.

[12] M. A. Akbar, N. H. M. Ali, Exp-function method for Duffing equation and new solutions of $(2+1)$ dimensional dispersive long wave equations, Prog. Appl. Math., 1 (2) (2011).

[13] H. Naher, A. F. Abdullah, M. A. Akbar, The Exp-function method for new exact solutions of the nonlinear partial differential equations, Int. J. Phys. Sci., 6 (29) (2011) 6706-6716.

[14] A. M. Wazwaz, A Applied sine-cosine method for handle nonlinear wave equations, Math. Comput. Modeling, 40 (2004) 499-508.

[15] N. Taghizadeh and M. Mirzazadeh, The first integral method to some complex nonlinear partial differential equations, J.Comput. Appl. Math., 235 (2011) 4871-4877.

[16] Y. Khan, H. Va' zquez-Leal, N. Faraz, An auxiliary parameter method using Adomian polynomials and Laplace transformation for nonlinear differential equations, Appl. Math. Model. 37 (2013) 2702-2708.

[17] J. Weiss, M. Tabor and G. Carnevale, The Painlevé property for partial differential equations, J. Math. Phys., 24 (1982) 522-526. 
[18] G. Adomian, Solving Frontier Problems of Physics: The Decomposition Method, Kluwer Academic, Boston, MA, 1994, Periodic.

[19] Z. Yan and H. Zhang, New explicit solitary wave solutions and periodic wave solutions for Whitham Broer-Kaup equation in shallow water, Physics Letters. A, 285 (5-6) (2001) 355-362.

[20] A. L. Guo and J. Lin, "Exact solutions of (2+1)-dimensional HNLS equation”, Commun. Theor. Phys., 54 (2010), pp. 401406.

[21] Y. He, S. Li, Y. Long, Exact solutions of the Klein-Gordon equation by modified Exp-function method, Int. Math. Forum, 7(4) (2012) 175-182.

[22] Biswas A, Zony C, Zerrad E. Soliton perturbation theory for the quadratic nonlinear Klein-Gordon equation. Appl Math Comput 2008; 203:153-6.

[23] Khan K, Akbar MA. 2014 Exact traveling wave solutions of Kadomtsev-Petviashvili equation. J. Egypt. Math. Soc. 23, 278-281. (doi:10.1016/j.joems.2014.03.010).

[24] K. Khan and M. A. Akbar, Application of $\exp (-\Phi(\eta))-$ expansion method to find the exact solutions of modified Benjamin-Bona-Mahony equation, World Appl. Sci. J., 24(10) 2013 1373-1377.

[25] R. Islam, M. N. Alam, A. K. M. K. Sazzad Hossain, H. O. Roshid, M. A. Akbar, Traveling wave solutions of nonlinear evolution equations via $\operatorname{Exp}(-\Phi(\eta))$-expansion method, Global J. Sci. Frontier Res., 13(11) (2013) 63-71.

[26] M. N. Alam, M. A. Akbar and H. O. Roshid, Study of nonlinear evolution equations to construct traveling wave solutions via the new approach of generalized $\left(G^{\prime} / G\right)$ expansion method, Math. Stat., 1(3) (2013) 102-112.
[27] M. A. Akbar, N. H. M. Ali, Exact Solutions to Some Nonlinear Partial Differential Equations in Mathematical Physics Via the $\left(\mathrm{G}^{\prime} / \mathrm{G}\right)$-Expansion Method, Research J. of App. Sciences, Engineering and Technology 6(19): 35273535,2013

[28] M. A. Akbar, N. H. M. Ali, E. M. E. Zayed, Abundant exact traveling wave solutions of the generalized Bretherton equation via $\left(\mathrm{G}^{\prime} / \mathrm{G}\right)$-expansion method, Commun. Theor. Phys., 57 (2012) 173-178.

[29] He JH. An elementary introduction to recently developed asymptotic methods and nano- mechanics in textile engineering. Int. J. Mod. Phys. B2008; 22 (21) 3487-578.

[30] Zhang, J., F. Jiang and X. Zhao, An improved $\left(\mathrm{G}^{\prime} / \mathrm{G}\right)$ expansion method for solving nonlinear evolution equations, Int. J. Com. Math., 87 (8) (2010) 1716-1725.

[31] J. Akter, M. A. Akbar, Exact solutions to the Benney-Luke equation and the Phi-4 equations by using modified simple equation method. Results in physics 5 (2015) 125-130.

[32] Md. Ashrafuzzaman Khan, M. Ali Akbar, Fethi Bin Muhammad Belgacem. Solitary Wave Solutions for the Boussinesq and Fisher Equations by the Modified Simple Equation Method. Mathematics Letters. 2 (1) (2016) 1-18.

[33] A. J. M. Jawad, M. D. Petkovic and A. Biswas, "Modified simple equation method for nonlinear evolution equations", Appl. Math. Comput., 217 (2010) 869-877.

[34] K. Khan, M. A. Akbar, Exact and solitary wave solutions for the Tzitzeica-Dodd-Bullough and the modified $\mathrm{KdV}$ Zakharov-Kuznetsov equations using the modified simple equation method, Ain Shams Eng. J., 4 (4) (2013) 903-909. 